\title{
Unscrambling Naoto Fukasawa's Design Concept "Without Thought" by the View of the Unconscious Psychology
}

\author{
Ying Deng \\ School of Design \& Art \\ University of South China \\ Hengyang, China 421001 \\ Academy of Arts \& Design \\ Tsinghua University \\ Beijing, China 100084
}

\author{
Jun Zhou* \\ School of Design \& Art \\ University of South China \\ Hengyang, China 421001 \\ *Corresponding Author
}

\begin{abstract}
Objective: It attempts to have a better understanding of Naoto Fukasawa's the concept "Without Thought" from the view of psychology. Method It finds the similarities and differences by comparing "Without Thought" and "unconscious" in psychology, and then discusses the psychological basis, core thought and key issue of "Without Thought". Finally, it unscrambles three works of Naoto Fukasawa. Conclusion The "unconscious" research results from cognitive psychology can be used as psychological basis of Naoto Fukasawa "Without Thought". Ecological view is the core thought of "Without Thought". It is the key issue that "Without Thought" means unconscious states and intermediate states of awareness. Reducing consciousness would affect the operation of unconscious mentality which could awaken core consciousness, by affording it more opportunity to control behavior.
\end{abstract}

Keywords-Without Thought; unconscious; Naoto Fukasawa; implicit memory

\section{INTRODUCTION}

Without Thought is an unconscious design workshop organized by Naoto Fukasawa in Japan DMN. It aims to explore design ideas from people's daily lives. Without Thought (usually translated as the unconscious) has become a label for Naoto Fukasawa's design. But it is difficult for the public to understand the concept of the unconscious, and it seems to be a mysterious word. In fact, the unconscious involves a wide range of subjects, such as psychology, philosophy, psychiatry, law, literature and art. But it has a long history and in-depth scientific research in the field of psychology. The unconscious has been discussed as early as Platon and Aristotle. Freud found that people were driven by the unknown and uncontrollable power, and he took the unconscious as object of study. Then Carl Jung, Lacan and Fromm also carried out their research on the theory of the unconscious. In the middle of the 20th Century, cognitive psychology began to study the unconscious. Although unconsciousness is still a controversial is sue in psychology, the unconscious research results in psychology will give us a broader understanding space. Therefore, it is a good way to interpret Naoto Fukasawa's unconscious design from psychology.

\section{THE PSYCHOLOGICAL BASIS OF NAOTO FUKASAWA'S CONCEPT OF "WITHOUT THOUGHT"}

The theory of psychological unconsciousness mainly comes from the two subjects, psychoanalysis and cognitive psychology. Unconscious research in psychoanalysis was kicked off from Freud, and now it is still main research field of cognitive psychology. Since the middle of 20th century, unconscious research has been extended emotion and psychology in skill, perception, attention, cognition, learning, memory and decision making. The two unconsciousness schools have significant differences in research methods, content and opinions. In a comprehensive comparison, Naoto Fukasawa's Without Thought is more close to the unconscious view of cognitive psychology.

Naoto Fukasawa participated in the design work in ID TWO and IDEO in the United States from 1989 to 1996. At that time, A merican was in the period of rapid development of computers, and the research and application of cognitive psychology in computer and design field were deepened. In 1997, he assisted and organized a branch of IDEO in Japan. In 1998, he organized "Without Thought" workshop in Japan and shared his ideas. From his experience, Naoto Fukasawa has been greatly enlightened and influenced by human-computer interaction of American cognitive psychology in his reflection of the relationship between human and things.

From the comparison of the unconscious interpretation, psychoanalytic scholars believe that behaviors of individual or group under specific cultural background, including both mental patients and normal people, are all driven by the unconscious in mentality. Corresponding to the driving function draws forth defense mechanism (a mental mechanism that control bad mental contents outside mental level and keeps the psychological balance). The unconscious research of cognitive psychology is unfolded with the study of consciousness. It does not approve the psychological defense 
mechanism and exaggerated unconscious drive. The unconscious in cognitive psychology refers to a conscious situation or level and the mental-processing mechanism under this level of consciousness. Naoto Fukasawa's Without Thought refers to the unthought state of people in everyday life. It also includes a level of consciousness (for instance, some small actions in daily life is sometimes completely unconscious or conscious situation (for example, cannot think of the shape of door handle after opening the door; the shape of door handle forms a unconscious or conscious situation) and explicit behavior under the level of consciousness. Naoto Fukasawa respects the cognitive fact of human beings, things and environment. He believes that it had nothing to do with human's emotion and moral quality. Therefore, he also doesn't approve the so-called defensive mechanism to eliminate bad psychological contents.

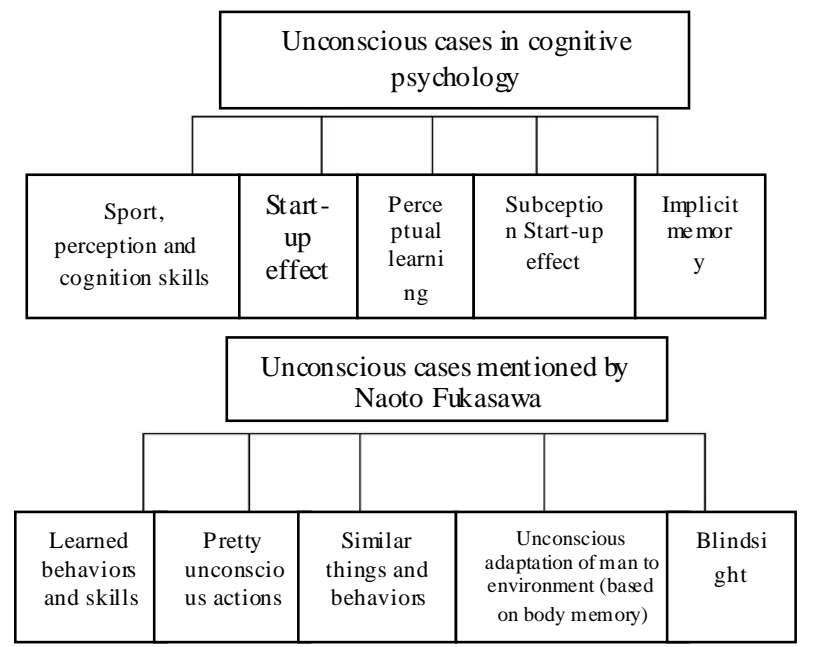

Fig. 1. Unconscious example comparison between cognitive psychology and Naoto Fukasawa's concept.

On the other hand, Naoto Fukasawa's Without Thought examples and his potential attitude also show cognitive unconscious tendency in "Fig. 1". Dancers and athletes... If they can't go beyond consciousness, they can't do it"[1]. Naoto Fukasawa talked about the acquisition of many such unconscious skills and behaviors which are the results of a lot of deliberate learning. The unconscious states on familiar things and behaviors in the daily life are results of a lot of unintended experience. The unconscious adaptation of man to environment is also based on past experience (once entering into a tiled-paved room without umbrella shelf, one will naturally lean his umbrella on the wall with sharp point putting on the gap of tiles [2]). These behaviors needn't to be deliberately recollected, and can be automatically controlled. Naoto Fukasawa thinks this is related to "active memory". He said that memory neither refers to recollection nor the memorable things in the brain, for they are easy to be thought of and associated. Memory refers to "memory described by body or all phenomena felt in unconscious level"[1]. Therefore, active memory is from the angle of cognitive psychological unconsciousness. It is not explicit memory (descriptive memory) that we usually perceive, but implicit memory (nondescriptive memory). Because of the unconsciousness of implicit memory, we often think that it doesn't belong to memory. Programmed memory belongs to implicit memory. It is a memory about doing something. Such memory can be acquired by many attempts. When using this kind of memory, it can be automatically invoked without the participation of consciousness. Several unconscious phenomena mentioned above need to be combined with the body. In practice, it is possible to complete the function of procedural memory. Naoto Fukasawa also mentioned an experiment: "let people stand in front of the wall, and then move the wall forward at two to three centimeters at a speed that is not detected, and these people will move along with it"[1]. It means that eyes do not see but "perceiving" things, and the body will still perceive the dynamic action of the surrounding environment and make the correct reflection. Also in cognitive psychology the experiments are also carried out, and they call it blind sight. It refers to phenomena that people cannot detect stimulation or change, but they can process it to in a certain degree. It results in the function of subliminal perception

Although Naoto Fukasawa's Without Thought is close to the cognitive unconsciousness, it is not so accurate to be completely understood by cognitive unconsciousness. The two sides are exploring the unconscious in different world outlooks, and their definitions on the unconscious are also different. The elaboration of these differences helps us better understand Naoto Fukasawa's Without Thought.

\section{NAOTO FUKASAWA'S ECOLOGICAL VIEW AND WITHOUT THOUGHT VIEW}

From Naoto Fukasawa's interpretation of design, we can see his ecological consciousness. He believes that human beings and things are part of the natural ecosystem. Things are the extension of the body and the extension of the ecosystem, and more accurately the extension of the relationship between man and the environment. Things are born in the potential relationship between man and the environment, for anything in the ecological circle are results of the balanced actions of internal and external forces. The external force refers to the impetus of external physical environment and spiritual environment (including self, because it is part of the environment). The internal force refers to the counterforce of internal spiritual power. So, this potential relationship has natural balance and flexibility. The elastic boundary between them is the contours of the existence of things. People's unthinking behavior is reflecting the natural relationship of environment all people share, and facing the changing of the environment, unconsciously adaptive behavior also reflects the dynamic interaction between human and environment in order to achieve a balanced state, which is a beautiful state.

Too many designs are now in pursuit of new and exciting effects, and designers try to make things more attractive. Naoto Fukasawa believes that if the designer make designs over deliberately or subjectively, or to meet the needs of clients indefinitely, the works will have strong tensile force, destructing the potential balance between man and environment. Thus it will disturb the order of ecosystem, excessively invade and occupy the nature. Then the ecosystem will be unstable and out of harmony. "If possible, we shall avoid too many forces in design, and eliminate shape that pushes the outline outward. What we really need is shapes that 
can balance internal and external forces" [1]. Based on this, Naoto Fukasawa emphasizes the importance of this potential relationship and the level of consciousness of unthought behavior.

Therefore, Naoto Fukasawa's concept is different from the concept of cognitive psychology which is centered by man and sees things at the perspective of human beings, and also different from concept of social cognition psychology which attaches importance to the influence of external situation on man. The unconscious of Naoto Fukasawa focuses on the internal psychological process of human beings. Naoto Fukasawa's Without Thought shall be understood in the context of ecological environ ment, for it takes the whole hu man beings as a group and abandons constraints of personal subjectivity, social norms and human morality, and has nothing to do with goodness and evil. So, Naoto Fukasawa's Without Thought view reflects apparent objectivity, universality and publicity.

\section{Without ThOUGHT PROPOSED BY NAOTO FUKASAWA IS NOT COMPLETELY UNCONSCIOUS}

It is not appropriate to interpret Naoto Fukasawa's Without Thought into the unconscious. Does Without Thought mean unconsciousness? First of all, we found that Naoto Fukasawa has used many similar terms, including without thinking, unconscious, not aware of, not thinking too much, unthinking(unthinkingly), intuitively aware (from DMA), in explaining Without Thought. Secondly, if we explore Naoto Fukasawa's unthought behaviors carefully, we will find that the behaviors he describes is out of both unconsciousness and consciousness. In addition, from the methods to determine the level of consciousness, cognitive experiment psychology mostly uses subjective report of testers (if the reports of testers says he didn't remember or perceive) and combines objective measurement methods (the influence of stimulation on subsequent behaviors by observation) to determine comprehensively. Naoto Fukasawa's Without Thought is based on experience. He advocates determining and obtaining information of unconscious behaviors by natural observation. Strictly speaking, it is not completely reliable. So, Without Thought proposed by Naoto Fukasawa is not completely unconscious.

To understand Naoto Fukasawa's conscious state of Without Thought behavior, maybe we can get enlightenment from the different viewpoints of cognitive psychology on the boundary of unconsciousness and consciousness. Cognitive psychologists have recognized that unconsciousness and consciousness are related to two different and interacting memory systems (implicit memory and explicit memory) respectively, and are also supported by different brain systems. But in recent years some psychologists have questioned the dual viewpoint of unconsciousness and consciousness. They put forward a hierarchy of consciousness. Except for pure unconsciousness and pure conscious ness, there is also a middle zone of consciousness. Thus, some behaviors can be regarded as unconscious behavior or low-level conscious behavior. But up to now, the study of cognitive psychology on the middle state of consciousness is still shallow. This impure unconsciousness is also mentioned in Freud's theory. That is preconsciousness. The present study of psychoanalysis also acknowledges the middle zone of consciousness which is called as the flow boundary between consciousness and unconsciousness. Without Thought behavior observed by Naoto Fukasawa includes not only pure unconscious phenomena, but also the middle zone of some consciousness and unconsciousness. It flows between unconsciousness and consciousness; sometimes is unconscious; sometimes it is lowlevel conscious. The influences of pure unconsciousness or the low-load attention consciousness are not very different to daily life behavior. Therefore, pure unconscious behavior is not the purpose of Naoto Fukasawa directly.

Behavior is completed by consciousness and unconsciousness together. Psychologists believe that in some cases, the two systems of consciousness and unconsciousness optimize behavior together. In some cases, the two systems compete, for a loss of one system will affect the operation of the other system, and give more room to control behavior. Naoto Fukasawa emphasizes the importance of Without Thought. He thinks too much self consciousness interferes the participation of unconsciousness, and might conflict with unconscious behavior, or even "surpass consciousness and get the maximum coordination with environment"[1]. Sasaki Masahito thinks that Naoto Fukasawa's Without Thought is "evoking the search for core consciousness"[2]. That is to say, they advocate that designers shouldn't be distracted by users, so as to leave more room for them to process core problem between the flow behavior of unconsciousness or low-level consciousness.

\section{ACTIVATION, AWAKENING AND FIT-NAOTO FUKASAWA'S DESIGN}

"The unconscious and harmonious relationship between human and things is the best expression of Without Thought design"[2]. In the following, some examples are listed to explain works of Naoto Fukasawa.

\section{A. Overlap and Connection of Behavior}

Multiple severed actions can be organized into a continuous action. Everything will run smoothly and naturally, which can reduce the participation of consciousness, and the unconscious behavior will come from it. This method is used in some of Naoto Fukasawa's designs, such as, the printer designed by Naoto Fukasawa in "Fig. 2". Naoto Fukasawa observed that people usually print at least two copies for comparison and select the best one, and throw the rest into the trash can. People did not notice that the two acts of printing and throwing wastepaper were closely connected in the office every day. Because of the separation state of objects, there appeared two completely severed behaviors. We have to look for a trash can cons ciously after using the printer. Co mbining printer and trash will eliminate the seeking action between the two behaviors and form a s mooth and unthought behavior process. The light with dish in "Fig. 3" also shows this concept of Naoto Fukasawa. He connects the series of actions of people after going home and before sleep - open light + put belongings; put glasses + close light. 


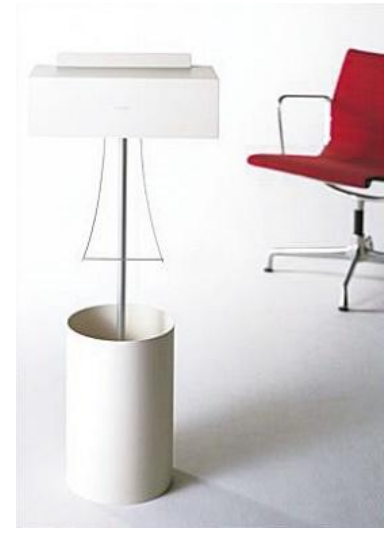

Fig. 2. Printer EPSON.

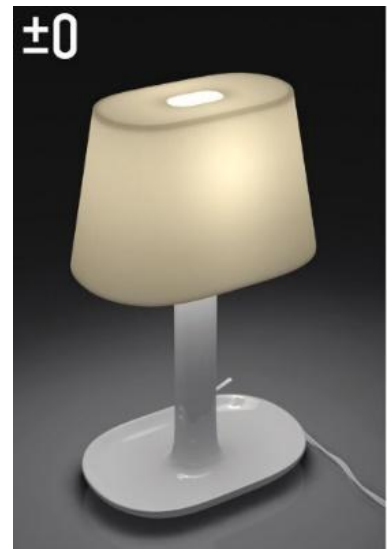

Fig. 3. Light with Dish \pm 0 .

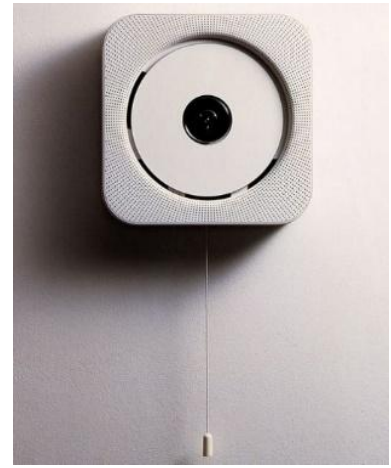

Fig. 4. CD Player for MUJI.

\section{B. Feeling/Memory Condensation}

Many people think that the CD player in "Fig. 4" designed by Naoto Fukasawa is more like a work of art. He overlapped the image of ventilating fan and CD player. It is similar to condensation which condenses a plurality of image into a comprehensive image. It is based on experience and memory, but does not reflect the true memory. It is a little like dreaming and not in accordance with the reality. It is an imagination. Freud believes that condensation is a way for unconsciousness rising to consciousness. In this design, the condensation method is used to create a dreamy scene which can wake up deep unconscious feelings and memories. From this point of view, people regard it an art than a simple product.

\section{Buried Unconscious Function}

In the environment and things, it is allowed to bury the unconscious function, which is a way of Naoto Fukasawa to present the unconsciousness. This approach provides a possible space for behavior, but it is not mandatory or catered to. Naoto Fukasawa looks for the potential relationship between man and the environment, subtly designs the elastic space, and maintains the potential relationship by free adjustment of human and environment. The pen box in "Fig. 5" he designed for $B \& B$ is a typical example of this. When we put the pen on desk after use, it is usually an optional behavior without thinking. Naoto Fukasawa designed many interlaced grooves on the box in order to fit the unconscious placement behaviors.

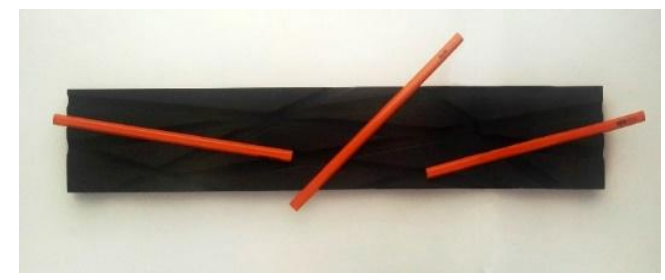

Fig. 5. Pen box for B\&B.

\section{CONCLUSION}

Naoto Fukasawa's Without Thought shows the unconscious tendency of cognitive psychology. He respects the objective potential relationship between human and environmental cognition, and affirms the role of implicit memory in balancing the relationship. Naoto Fukasawa's concept of Without Thought is not centered on human beings, but is understood in the broad vision of the ecosystem. It is the designer's cognition and reflection on design. His works and planning activities are also his practice of Without Thought concept. Naoto Fukasawa not only emphasizes the unconscious ness, but also cares about the elastic boundary between consciousness and the unconsciousness. Looking for them and presenting them properly are Naoto Fukasawa's Without Thought design concept.

\section{REFERENCES}

[1] Takeshi Goto, Masato Sasaki, Naoto Fukasawa, The Ecological Approach to Design, 1st ed., Guilin: Guangxi normal university press, March 2016.

[2] Bill Moggridge , Tim Brown, Kenya Hara, Antony Gormley Studio, Naoto Fukasawa. London: Phaidon Press Ltd, 1 st ed., July 2013.

[3] Larry R. Squire, Adam J. Dede . "Conscious and Unconscious Memory Systems," Cold Spring Harbor Perspectives in Biology, 2015.

[4] Freud S., Unconscious, Beijing: Sailing Culture Communication Co., Ltd., 2016.

[5] Freud S., The Ego and Id, Shanghai: Shanghai Translation Publishing House, 2011.

[6] Jung C.G., Jung's Collected Works, Beijing: The Reform Press, 1997.

[7] Ron L.F., From Freud to Carl Jung - Comparative Study on the Psychology of the Unconscious, Beijing: International Broadcasting Press of China, 1989. 
[8] Cheng Juiqing, "A Review of the Unconscious in Cognitive Psychology - the Unconscious Comparison Between Cognitive Psychology and Psychoanalysis," Academic Journal of Suzhou University(Philosophy and Social Science), pp. 113-116, 2005,(02).

[9] Norman E., "The Unconscious" in Current Psychology," European Psychologist, pp. 193-201, 2010. 15(3)

[10] Powen L.A. Personality Science, Shanghai: East China Normal University Press, 2001

[11] Newell B. R., Shanks D. R., "Unconscious Influences on Decision Making: a Critical Review," Behavioral and Brain Science, 2014(37)

[12] Berridge K., Winkielman P., "What is an Unconscious Emotion? (The Case for Unconscious Liking)," Cognition and Emotion, 2003

[13] Epstein S., "Demystifying Intuition: What It Is, What It Does, and How It Does It,"P sychological Inquiry, 2010. 